\title{
A Genus-3 Topological Recursion Relation
}

\author{
Takashi Kimura $\stackrel{*}{,}$ Xiaobo Liu ${ }^{\dagger}$
}

\begin{abstract}
In this paper, we give a new genus-3 topological recursion relation for GromovWitten invariants of compact symplectic manifolds. This formula also applies to intersection numbers on moduli spaces of spin curves. A by-product of the proof of this formula is a new relation in the tautological ring of the moduli space of 1-pointed genus-3 stable curves.
\end{abstract}

Let $\overline{\mathcal{M}}_{g, n}$ be the moduli space of genus- $g$ stable curves with $n$ marked points. It is well known that relations in the tautological rings on $\overline{\mathcal{M}}_{g, n}$ produce universal equations for the Gromov-Witten invariants of compact symplectic manifolds. Examples of genus-1 and genus-2 universal equations were given in [Ge1, Ge2, and [BP. Relations among known universal equations were discussed in [L2]. It is expected that for manifolds with semisimple quantum cohomology, such universal equations completely determine all higher genus Gromov-Witten invariants in terms of its genus-0 invariants. This has been proven for the genus-1 case in [DZ] and for the genus-2 case in [L1]. However for genus bigger than 2, no explicit universal equations had been found except for those which follow from an obvious dimension count. The main purpose of this paper is to introduce a new genus-3 universal equation, namely, a genus-3 topological recursion relation.

Associated to the Gromov-Witten invariants of a compact symplectic manifold $M$ is its big phase space, a product of infinitely many copies of $H^{*}(M ; \mathbb{C})$. We will choose a basis $\left\{\gamma_{\alpha} \mid \alpha=1, \ldots, N\right\}$ of $H^{*}(M ; \mathbb{C})$. The quantum product $\mathcal{W}_{1} \circ \mathcal{W}_{2}$ of two vector fields $\mathcal{W}_{1}$ and $\mathcal{W}_{2}$ on the big phase space was introduced in [L1]. This is an associative product without an identity element. An operator $T$ on the space of vector fields on the big phase space was also introduced in [L1] to measure the failure of the string vector field to be an identity element with respect to this product. This operator turns out to be a very useful device to translate relations in the tautological rings of $\overline{\mathcal{M}}_{g, n}$ into universal equations for Gromov-Witten invariants. We will write universal equations of Gromov-Witten invariants as equations among tensors $\left\langle\left\langle\mathcal{W}_{1} \cdots \mathcal{W}_{k}\right\rangle_{g}\right.$ which are defined to be the $k$-th covariant derivatives of the generating functions of genus- $g$ Gromov-Witten invariants with respect to the trivial connection on the big phase space. We will briefly review these definitions in Section 1 for completeness.

The main result of this paper is the following theorem.

\footnotetext{
${ }^{*}$ Research of the first author was partially supported by NSF grant DMS-0204824.

${ }^{\dagger}$ Research of the second author was partially supported by NSF grant DMS-0505835.
} 
Theorem 0.1 For Gromov-Witten invariants of any compact symplectic manifold, the following topological recursion relation holds for any vector field $\mathcal{W}$ on the big phase space:

$$
\begin{aligned}
& \left\langle\left\langle T^{3}(\mathcal{W})\right\rangle\right\rangle_{3} \\
& =-\frac{1}{252}\left\langle\left\langle\mathcal{W} T\left(\gamma_{\alpha} \circ \gamma^{\alpha}\right)\right\rangle_{2}+\frac{5}{42}\left\langle\left\langle T\left(\gamma_{\alpha}\right)\left\{\mathcal{W} \circ \gamma^{\alpha}\right\}\right\rangle\right\rangle_{2}\right. \\
& +\frac{13}{168}\left\langle\left\langle T\left(\gamma^{\alpha}\right)\right\rangle\right\rangle_{2}\left\langle\left\langle\gamma_{\alpha} \mathcal{W} \gamma^{\beta} \gamma_{\beta}\right\rangle\right\rangle_{0}+\frac{41}{21}\left\langle\left\langle T\left(\gamma^{\alpha}\right)\right\rangle\right\rangle_{2}\left\langle\left\langle\left\{\gamma_{\alpha} \circ \mathcal{W}\right\}\right\rangle\right\rangle_{1} \\
& -\frac{13}{168}\left\langle\left\langle\left\{\mathcal{W} \circ \gamma_{\alpha} \circ \gamma^{\alpha}\right\}\right\rangle_{2}+\frac{1}{280}\left\langle\left\langle\mathcal{W} \gamma^{\alpha}\right\rangle_{1}\left\langle\left\langle\gamma_{\alpha}\left\{\gamma^{\beta} \circ \gamma_{\beta}\right\}\right\rangle\right\rangle_{1}\right.\right. \\
& -\frac{23}{5040}\left\langle\left\langle\gamma^{\alpha}\right\rangle\right\rangle_{1}\left\langle\left\langle\gamma_{\alpha} \mathcal{W}\left\{\gamma^{\beta} \circ \gamma_{\beta}\right\}\right\rangle\right\rangle_{1}-\frac{47}{5040}\left\langle\left\langle\gamma^{\alpha}\right\rangle\right\rangle_{1}\left\langle\left\langle\gamma_{\alpha} \gamma^{\beta}\right\rangle\right\rangle_{1}\left\langle\left\langle\gamma_{\beta} \mathcal{W} \gamma^{\mu} \gamma_{\mu}\right\rangle\right\rangle_{0} \\
& -\frac{5}{1008}\left\langle\left\langle\mathcal{W} \gamma^{\alpha}\right\rangle\right\rangle_{1}\left\langle\left\langle\gamma_{\alpha} \gamma^{\beta} \gamma_{\beta} \gamma^{\mu}\right\rangle\right\rangle_{0}\left\langle\left\langle\gamma_{\mu}\right\rangle\right\rangle_{1}+\frac{23}{504}\left\langle\left\langle\gamma^{\alpha}\right\rangle\right\rangle_{1}\left\langle\left\langle\gamma_{\alpha} \mathcal{W} \gamma^{\beta} \gamma_{\beta} \gamma^{\mu}\right\rangle\right\rangle_{0}\left\langle\left\langle\gamma_{\mu}\right\rangle\right\rangle_{1} \\
& +\frac{11}{140}\left\langle\left\langle\gamma^{\alpha} \gamma^{\beta}\right\rangle\right\rangle_{1}\left\langle\left\langle\gamma_{\alpha}\left\{\gamma_{\beta} \circ \mathcal{W}\right\}\right\rangle_{1}-\frac{4}{35}\left\langle\left\langle\gamma^{\alpha}\right\rangle\right\rangle_{1}\left\langle\left\langle\gamma_{\alpha} \gamma^{\beta}\right\rangle\right\rangle_{1}\left\langle\left\langle\left\{\gamma_{\beta} \circ \mathcal{W}\right\}\right\rangle\right\rangle_{1}\right. \\
& +\frac{2}{105}\left\langle\left\langle\mathcal{W} \gamma^{\alpha}\right\rangle\right\rangle_{1}\left\langle\left\langle\left\{\gamma_{\alpha} \circ \gamma_{\beta}\right\}\right\rangle\right\rangle_{1}\left\langle\left\langle\gamma^{\beta}\right\rangle\right\rangle_{1}+\frac{89}{210}\left\langle\left\langle\gamma^{\alpha}\right\rangle\right\rangle_{1}\left\langle\left\langle\gamma_{\alpha} \mathcal{W} \gamma^{\beta} \gamma^{\mu}\right\rangle\right\rangle_{0}\left\langle\left\langle\gamma_{\beta}\right\rangle\right\rangle_{1}\left\langle\left\langle\gamma_{\mu}\right\rangle\right\rangle_{1} \\
& -\frac{1}{210}\left\langle\left\langle\gamma^{\alpha}\right\rangle\right\rangle_{1}\left\langle\left\langle\gamma_{\alpha} \gamma^{\beta}\left\{\gamma_{\beta} \circ \mathcal{W}\right\}\right\rangle\right\rangle_{1}+\frac{1}{140}\left\langle\left\langle\mathcal{W} \gamma^{\alpha} \gamma^{\beta}\right\rangle\right\rangle_{1}\left\langle\left\langle\left\{\gamma_{\alpha} \circ \gamma_{\beta}\right\}\right\rangle\right\rangle_{1} \\
& +\frac{23}{140}\left\langle\left\langle\gamma^{\alpha} \gamma^{\beta}\right\rangle\right\rangle_{1}\left\langle\langle \gamma _ { \alpha } \gamma _ { \beta } \mathcal { W } \gamma ^ { \mu } \rangle _ { 0 } \left\langle\left\langle\gamma_{\mu}\right\rangle_{1}-\frac{3}{140}\left\langle\left\langle\gamma^{\alpha} \gamma^{\beta}\right\rangle\right\rangle_{1}\left\langle\left\langle\left\{\gamma_{\alpha} \circ \gamma_{\beta}\right\} \mathcal{W}\right\rangle_{1}\right.\right.\right. \\
& -\frac{1}{4480}\left\langle\left\langle\mathcal{W} \gamma^{\alpha}\right\rangle\right\rangle_{1}\left\langle\left\langle\gamma_{\alpha} \gamma_{\beta} \gamma^{\beta} \gamma_{\mu} \gamma^{\mu}\right\rangle\right\rangle_{0}+\frac{13}{8064}\left\langle\left\langle\gamma^{\alpha}\right\rangle\right\rangle_{1}\left\langle\left\langle\gamma_{\alpha} \mathcal{W} \gamma^{\beta} \gamma_{\beta} \gamma^{\mu} \gamma_{\mu}\right\rangle\right\rangle_{0} \\
& -\frac{1}{2240}\left\langle\left\langle\mathcal{W} \gamma^{\alpha} \gamma^{\beta}\right\rangle\right\rangle_{1}\left\langle\left\langle\gamma_{\alpha} \gamma_{\beta} \gamma^{\mu} \gamma_{\mu}\right\rangle_{0}+\frac{41}{6720}\left\langle\left\langle\gamma^{\alpha} \gamma^{\beta}\right\rangle\right\rangle_{1}\left\langle\left\langle\gamma_{\alpha} \gamma_{\beta} \mathcal{W} \gamma^{\mu} \gamma_{\mu}\right\rangle\right\rangle_{0}\right. \\
& +\frac{1}{53760}\left\langle\left\langle\mathcal{W} \gamma^{\alpha} \gamma_{\alpha} \gamma^{\beta} \gamma_{\beta} \gamma^{\mu} \gamma_{\mu}\right\rangle\right\rangle_{0}-\frac{1}{210}\left\langle\left\langle\left\{\mathcal{W} \circ \gamma^{\alpha}\right\}\right\rangle_{1}\left\langle\left\langle\gamma_{\alpha} \gamma^{\beta} \gamma_{\beta}\right\rangle\right\rangle_{1}\right. \\
& -\frac{1}{5760}\left\langle\left\langle\mathcal{W} \gamma^{\alpha} \gamma_{\alpha}\left\{\gamma^{\beta} \circ \gamma_{\beta}\right\}\right\rangle\right\rangle_{1}-\frac{1}{2688}\left\langle\left\langle\gamma^{\alpha} \gamma_{\alpha} \gamma^{\beta}\right\rangle\right\rangle_{1}\left\langle\left\langle\gamma_{\beta} \mathcal{W} \gamma^{\mu} \gamma_{\mu}\right\rangle\right\rangle_{0} \\
& -\frac{1}{5040}\left\langle\left\langle\gamma^{\alpha} \gamma_{\alpha} \gamma^{\beta}\left\{\gamma_{\beta} \circ \mathcal{W}\right\}\right\rangle\right\rangle_{1}+\frac{1}{3780}\left\langle\left\langle\mathcal{W} \gamma_{\alpha} \gamma_{\beta} \gamma_{\mu}\right\rangle\right\rangle_{1}\left\langle\left\langle\gamma^{\alpha} \gamma^{\beta} \gamma^{\mu}\right\rangle\right\rangle_{0} \\
& +\frac{1}{252}\left\langle\left\langle\gamma_{\alpha} \gamma_{\beta} \gamma_{\mu}\right\rangle\right\rangle_{1}\left\langle\left\langle\mathcal{W} \gamma^{\alpha} \gamma^{\beta} \gamma^{\mu}\right\rangle\right\rangle_{0} .
\end{aligned}
$$

A by-product of the proof of this theorem is a new relation in the tautological ring of $\overline{\mathcal{M}}_{3,1}$ which will be given in Section 3. This relation is equivalent to the genus-3 topological recursion relation.

The main idea behind the proof of Theorem 0.1 is that universal equations for GromovWitten invariants can be written as linear combinations of finitely many terms of a given dimension each corresponding to a boundary stratum, suitably decorated, in $\overline{\mathcal{M}}_{g, n}$. The goal is to determine the coefficients in these linear combinations. Suppose that all of the Gromov-Witten invariants of a particular manifold are known then since these invariants must satisfy the universal relations, it will imply relations between these coefficients.

In the case of our genus-3 topological recursion relation, the Gromov-Witten invariants 
of a point and of $\mathbb{C} P^{1}$, both of which are complete known, completely determine the coefficients and, hence, this universal relation. This method may be adapted to obtain more universal equations of any genus and will be explored in a future paper.

Finally, we observe that these universal equations not only apply to Gromov-Witten theory but also to other cohomological field theories, in the sense of Kontsevich-Manin, such as $r$-spin theory [JKV] where $r \geq 2$ is an integer. The correlators in $r$-spin theory are intersection numbers on the moduli space of $r$-spin curves, (cf. [JKV]). $r$-spin theory is interesting because of the generalized Witten conjecture which states that its big phase space potential function solves the $r$-th $\mathrm{KdV}$ integrable hierarchy. When $r=2$, this reduces to the ordinary Witten conjecture [W] proven by Kontsevich [K]. While this conjecture has been proven in special cases for general $r$ in low genus, it is still open in general. Since equation (11) also applies to $r$-spin theory, we use it to calculate some correlators in this theory.

After the completion of this paper, the preprint AL appeared in which a topological recursion relation equivalent to ours on $\overline{\mathcal{M}}_{3,1}$ was obtained assuming that the so-called invariance conjectures hold. However, since these conjectures have not yet been established, their work does not yet prove that our topological recursion relation holds on $\overline{\mathcal{M}}_{3,1}$.

Acknowledgments. The authors would like to thank Andreas Gathmann for allowing them to use his computer program for computing Gromov-Witten invariants of $\mathbb{C} P^{1}$ based

on the Virasoro constraints. We would also like to thank the Institut des Hautes Études Scientifiques for their hospitality and financial support where this paper was initiated, and the Focus on Mathematics Program at Boston University for the usage of their main server whether they are aware of it or not.

\section{Preliminaries}

Let $M$ be a compact symplectic manifold. The big phase space is by definition the infinite product

$$
P:=\prod_{n=0}^{\infty} H^{*}(M ; \mathbb{C}) .
$$

Fix a basis $\left\{\gamma_{0}, \ldots, \gamma_{N}\right\}$ of $H^{*}(M ; \mathbb{C})$, where $\gamma_{0}$ is the identity element, of the ordinary cohomology ring of $M$. Then we denote the corresponding basis for the $n$-th copy of $H^{*}(M ; \mathbb{C})$ in $P$ by $\left\{\tau_{n}\left(\gamma_{0}\right), \ldots, \tau_{n}\left(\gamma_{N}\right)\right\}$. We call $\tau_{n}\left(\gamma_{\alpha}\right)$ a descendant of $\gamma_{\alpha}$ with descendant level $n$. We can think of $P$ as an infinite dimensional vector space with a basis $\left\{\tau_{n}\left(\gamma_{\alpha}\right) \mid\right.$ $\left.0 \leq \alpha \leq N, n \in \mathbb{Z}_{\geq 0}\right\}$ where $\mathbb{Z}_{\geq 0}=\{n \in \mathbb{Z} \mid n \geq 0\}$. Let $\left(t_{n}^{\alpha} \mid 0 \leq \alpha \leq N, n \in \mathbb{Z}_{\geq 0}\right)$ be the corresponding coordinate system on $P$. For convenience, we identify $\tau_{n}\left(\gamma_{\alpha}\right)$ with the coordinate vector field $\frac{\partial}{\partial t_{n}^{\alpha}}$ on $P$ for $n \geq 0$. If $n<0, \tau_{n}\left(\gamma_{\alpha}\right)$ is understood to be the 0 vector field. We also abbreviate $\tau_{0}\left(\gamma_{\alpha}\right)$ by $\gamma_{\alpha}$. We use $\tau_{+}$and $\tau_{-}$to denote the operators which shift the level of descendants by 1 , i.e.

$$
\tau_{ \pm}\left(\sum_{n, \alpha} f_{n, \alpha} \tau_{n}\left(\gamma_{\alpha}\right)\right)=\sum_{n, \alpha} f_{n, \alpha} \tau_{n \pm 1}\left(\gamma_{\alpha}\right)
$$


where $f_{n, \alpha}$ are functions on the big phase space.

We will adopt the following notational conventions: Lower case Greek letters, e.g. $\alpha$, $\beta, \mu, \nu, \sigma, \ldots$, etc., will be used to index the cohomology classes on $M$. These indices run from 0 to $N$. Lower case English letters, e.g. $i, j, k, m, n, \ldots$, etc., will be used to index the level of descendants. These indices run over the set of all non-negative integers, i.e. $\mathbb{Z}_{\geq 0}$. All summations are over the entire ranges of the corresponding indices unless otherwise indicated. Let

$$
\eta_{\alpha \beta}=\int_{M} \gamma_{\alpha} \cup \gamma_{\beta}
$$

be the intersection form on $H^{*}(M, \mathbb{C})$. We will use $\eta=\left(\eta_{\alpha \beta}\right)$ and $\eta^{-1}=\left(\eta^{\alpha \beta}\right)$ to lower and raise indices. For example,

$$
\gamma^{\alpha}:=\eta^{\alpha \beta} \gamma_{\beta}
$$

Here we are using the summation convention that repeated indices (in this formula, $\beta$ ) should be summed over their entire ranges.

Let

$$
\left\langle\tau_{n_{1}}\left(\gamma_{\alpha_{1}}\right) \tau_{n_{2}}\left(\gamma_{\alpha_{2}}\right) \ldots \tau_{n_{k}}\left(\gamma_{\alpha_{k}}\right)\right\rangle_{g, d}:=\int_{\left[\overline{\mathcal{M}}_{g, n}(M ; d)\right]^{\mathrm{virt}}} \bigcup_{i=1}^{k}\left(\Psi^{n_{i}} \cup \mathrm{ev}_{\mathrm{i}}{ }^{*} \gamma_{\alpha_{i}}\right)
$$

be the genus- $g$, degree $d$, descendant Gromov-Witten invariant associated to $\gamma_{\alpha_{1}}, \ldots, \gamma_{\alpha_{k}}$ and nonnegative integers $n_{1}, \ldots, n_{k}$ (cf. [W], [RT], [LiT]). Here, $\overline{\mathcal{M}}_{g, k}(M ; d)$ is the moduli space of stable maps from genus- $g, k$-pointed curves to $M$ of degree $d \in H_{2}(M ; \mathbb{Z}) . \Psi_{i}$ is the first Chern class of the tautological line bundle over $\overline{\mathcal{M}}_{g, k}(M ; d)$ whose geometric fiber over a stable map is the cotangent space of the domain curve at the $i$-th marked point while $\mathrm{ev}_{\mathrm{i}}: \overline{\mathcal{M}}_{g, n}(M ; d) \rightarrow M$ is the $i$-th evaluation map for all $i=1, \ldots, k$. Finally, $\left[\overline{\mathcal{M}}_{g, n}(M ; d)\right]^{\text {virt }}$ is the virtual fundamental class. The genus- $g$ generating function is defined to be

$$
F_{g}=\sum_{k \geq 0} \frac{1}{k !} \sum_{\substack{\alpha_{1}, \ldots, \alpha_{k} \\ n_{1}, \ldots, n_{k}}} t_{n_{1}}^{\alpha_{1}} \ldots t_{n_{k}}^{\alpha_{k}} \sum_{d \in H_{2}(V, \mathbb{Z})} q^{d}\left\langle\tau_{n_{1}}\left(\gamma_{\alpha_{1}}\right) \tau_{n_{2}}\left(\gamma_{\alpha_{2}}\right) \ldots \tau_{n_{k}}\left(\gamma_{\alpha_{k}}\right)\right\rangle_{g, d}
$$

where $q^{d}$ belongs to the Novikov ring. This function is understood as a formal power series $\mathrm{n}$ the variables $\left\{t_{n}^{\alpha}\right\}$ with coefficients in the Novikov ring.

Introduce a $k$-tensor $\langle\langle\underbrace{\ldots \ldots}_{k}\rangle\rangle$ defined by

$$
\left\langle\left\langle\mathcal{W}_{1} \mathcal{W}_{2} \cdots \mathcal{W}_{k}\right\rangle\right\rangle_{g}:=\sum_{m_{1}, \alpha_{1}, \ldots, m_{k}, \alpha_{k}} f_{m_{1}, \alpha_{1}}^{1} \cdots f_{m_{k}, \alpha_{k}}^{k} \frac{\partial^{k}}{\partial t_{m_{1}}^{\alpha_{1}} \partial t_{m_{k}}^{\alpha_{2}} \cdots \partial t_{m_{k}}^{\alpha_{k}}} F_{g},
$$

for vector fields $\mathcal{W}_{i}=\sum_{m, \alpha} f_{m, \alpha}^{i} \frac{\partial}{\partial t_{m}^{\alpha}}$ where $f_{m, \alpha}^{i}$ are functions on the big phase space. This tensor is called the $k$-point (correlation) function. 
For any vector fields $\mathcal{W}_{1}$ and $\mathcal{W}_{2}$ on the big phase space, the quantum product of $\mathcal{W}_{1}$ and $\mathcal{W}_{2}$ is defined by

$$
\mathcal{W}_{1} \circ \mathcal{W}_{2}:=\left\langle\left\langle\mathcal{W}_{1} \mathcal{W}_{2} \gamma^{\alpha}\right\rangle\right\rangle_{0} \gamma_{\alpha}
$$

Define the vector field

$$
T(\mathcal{W}):=\tau_{+}(\mathcal{W})-\left\langle\left\langle\mathcal{W} \gamma^{\alpha}\right\rangle\right\rangle_{0} \gamma_{\alpha}
$$

for any vector field $\mathcal{W}$. The operator $T$ was introduced in [L1] as a convenient tool in the study of universal equations for Gromov-Witten invariants. Let $\psi_{i}$ be the first Chern class of the tautological line bundle over $\overline{\mathcal{M}}_{g, k}$ whose geometric fiber over a stable curve is the cotangent space of the curve at the $i$-th marked point. When we translate a relation in the tautological ring of $\overline{\mathcal{M}}_{g, k}$ into differential equations for generating functions of Gromov-Witten invariants, each $\psi$ class corresponds to the insertion of the operator $T$. Let $\nabla$ be the trivial flat connection on the big phase space with respect to which $\tau_{n}\left(\gamma_{\alpha}\right)$ are parallel vector fields for all $\alpha$ and $n$. Then the covariant derivative of the quantum product satisfies

$$
\nabla_{\mathcal{W}_{3}}\left(\mathcal{W}_{1} \circ \mathcal{W}_{2}\right)=\left(\nabla_{\mathcal{W}_{3}} \mathcal{W}_{1}\right) \circ \mathcal{W}_{2}+\mathcal{W}_{1} \circ\left(\nabla_{\mathcal{W}_{3}} \mathcal{W}_{2}\right)+\left\langle\left\langle\mathcal{W}_{1} \mathcal{W}_{2} \mathcal{W}_{3} \gamma^{\alpha}\right\rangle_{0} \gamma_{\alpha}\right.
$$

and the covariant derivative of the operator $T$ is given by

$$
\nabla_{\mathcal{W}_{2}} T\left(\mathcal{W}_{1}\right)=T\left(\nabla_{\mathcal{W}_{2}} \mathcal{W}_{1}\right)-\mathcal{W}_{2} \circ \mathcal{W}_{1}
$$

for any vector fields $\mathcal{W}_{1}, \mathcal{W}_{2}$ and $\mathcal{W}_{3}$ (cf. [L1, Equation (8) and Lemma 1.5]). We need to use these formulas in order to compute derivatives of universal equations.

\section{Proof of the genus-3 topological recursion relation}

The cohomology class $\psi_{1}^{g}$ vanishes on $\mathcal{M}_{g, 1}$ due to a result of Ionel (cf. [I]). It was proven in [GV] and [I] that $\psi_{1}^{g}$ on $\overline{\mathcal{M}}_{g, 1}$ is supported on the locus of curves which has at least one genus-0 component. Furthermore, by a result of Faber and Pandharipande [FP], $\psi_{1}^{g}$ is equal to a class from the boundary strata which is tautological, and therefore is a linear combination of products of $\psi$ and $\kappa$ classes and fundamental classes of some boundary strata. For $g=3, \kappa$ classes do not occur in this linear combination since components of curves in the boundary strata have genus at most 2 and $\kappa_{1}$ can be represented as linear combinations of $\psi$ classes and fundamental classes of boundary strata on the moduli spaces of stable curves of genus less than or equal to 2 (cf. [AC]). Therefore, it follows that $\psi_{1}^{3}$ on $\overline{\mathcal{M}}_{3,1}$ can be written as a linear combination of products of the $\psi$ classes and the fundamental classes of some boundary strata. By taking into consideration the genus0 and genus-1 topological recursion relations as well as Mumford's genus- 2 relation, we can translate these results into the following universal equations with unknown constants $a_{1}, \ldots, a_{30}$ :

$$
\begin{aligned}
0 & =\Phi(\mathcal{W}) \\
& :=-\left\langle\left\langle T^{3}(\mathcal{W})\right\rangle\right\rangle_{3}
\end{aligned}
$$




$$
\begin{aligned}
& +a_{1}\left\langle\left\langle T(\mathcal{W})\left\{\gamma_{\alpha} \circ \gamma^{\alpha}\right\}\right\rangle\right\rangle_{2}+a_{2}\left\langle\left\langle\mathcal{W} T\left(\gamma_{\alpha} \circ \gamma^{\alpha}\right)\right\rangle_{2}\right. \\
& +a_{3}\left\langle\left\langle T\left(\gamma^{\alpha}\right)\right\rangle\right\rangle_{2}\left\langle\left\langle\gamma_{\alpha} \mathcal{W} \gamma^{\beta} \gamma_{\beta}\right\rangle\right\rangle_{0}+a_{4}\left\langle\left\langle T\left(\gamma^{\alpha}\right)\right\rangle\right\rangle_{2}\left\langle\left\langle\left\{\gamma_{\alpha} \circ \mathcal{W}\right\}\right\rangle\right\rangle_{1} \\
& +a_{5}\left\langle\left\langle\left\{\mathcal{W} \circ \gamma_{\alpha} \circ \gamma^{\alpha}\right\}\right\rangle_{2}+a_{6}\left\langle\left\langle\mathcal{W} \gamma^{\alpha}\right\rangle_{1}\left\langle\left\langle\gamma_{\alpha}\left\{\gamma^{\beta} \circ \gamma_{\beta}\right\}\right\rangle\right\rangle_{1}\right.\right. \\
& +a_{7}\left\langle\left\langle\gamma^{\alpha}\right\rangle\right\rangle_{1}\left\langle\left\langle\gamma_{\alpha} \mathcal{W}\left\{\gamma^{\beta} \circ \gamma_{\beta}\right\}\right\rangle\right\rangle_{1}+a_{8}\left\langle\left\langle\gamma^{\alpha}\right\rangle\right\rangle_{1}\left\langle\left\langle\gamma_{\alpha} \gamma^{\beta}\right\rangle\right\rangle_{1}\left\langle\left\langle\gamma_{\beta} \mathcal{W} \gamma^{\mu} \gamma_{\mu}\right\rangle\right\rangle_{0} \\
& +a_{9}\left\langle\left\langle\mathcal{W} \gamma^{\alpha}\right\rangle\right\rangle_{1}\left\langle\left\langle\gamma_{\alpha} \gamma^{\beta} \gamma_{\beta} \gamma^{\mu}\right\rangle\right\rangle_{0}\left\langle\left\langle\gamma_{\mu}\right\rangle\right\rangle_{1} \\
& +a_{10}\left\langle\left\langle\gamma^{\alpha}\right\rangle\right\rangle_{1}\left\langle\left\langle\gamma_{\alpha} \mathcal{W} \gamma^{\beta} \gamma_{\beta} \gamma^{\mu}\right\rangle\right\rangle_{0}\left\langle\left\langle\gamma_{\mu}\right\rangle\right\rangle_{1}+a_{11}\left\langle\left\langle\gamma^{\alpha} \gamma^{\beta}\right\rangle\right\rangle_{1}\left\langle\left\langle\gamma_{\alpha}\left\{\gamma_{\beta} \circ \mathcal{W}\right\}\right\rangle_{1}\right. \\
& +a_{12}\left\langle\left\langle\gamma^{\alpha}\right\rangle\right\rangle_{1}\left\langle\left\langle\gamma_{\alpha} \gamma^{\beta}\right\rangle\right\rangle_{1}\left\langle\left\langle\left\{\gamma_{\beta} \circ \mathcal{W}\right\}\right\rangle\right\rangle_{1} \\
& +a_{13}\left\langle\left\langle\mathcal{W} \gamma^{\alpha}\right\rangle\right\rangle_{1}\left\langle\left\langle\left\{\gamma_{\alpha} \circ \gamma_{\beta}\right\}\right\rangle\right\rangle_{1}\left\langle\left\langle\gamma^{\beta}\right\rangle\right\rangle_{1} \\
& +a_{14}\left\langle\left\langle\gamma^{\alpha}\right\rangle\right\rangle_{1}\left\langle\left\langle\gamma_{\alpha} \mathcal{W} \gamma^{\beta} \gamma^{\mu}\right\rangle\right\rangle_{0}\left\langle\left\langle\gamma_{\beta}\right\rangle\right\rangle_{1}\left\langle\left\langle\gamma_{\mu}\right\rangle\right\rangle_{1} \\
& +a_{15}\left\langle\left\langle\gamma^{\alpha}\right\rangle\right\rangle_{1}\left\langle\left\langle\gamma_{\alpha} \gamma^{\beta}\left\{\gamma_{\beta} \circ \mathcal{W}\right\}\right\rangle\right\rangle_{1}+a_{16}\left\langle\left\langle\mathcal{W} \gamma^{\alpha} \gamma^{\beta}\right\rangle\right\rangle_{1}\left\langle\left\langle\left\{\gamma_{\alpha} \circ \gamma_{\beta}\right\}\right\rangle\right\rangle_{1} \\
& +a_{17}\left\langle\left\langle\gamma^{\alpha} \gamma^{\beta}\right\rangle\right\rangle_{1}\left\langle\left\langle\gamma_{\alpha} \gamma_{\beta} \mathcal{W} \gamma^{\mu}\right\rangle\right\rangle_{0}\left\langle\left\langle\gamma_{\mu}\right\rangle\right\rangle_{1}+a_{18}\left\langle\left\langle\gamma^{\alpha} \gamma^{\beta}\right\rangle\right\rangle_{1}\left\langle\left\langle\left\{\gamma_{\alpha} \circ \gamma_{\beta}\right\} \mathcal{W}\right\rangle\right\rangle_{1} \\
& +a_{19}\left\langle\left\langle\mathcal{W} \gamma^{\alpha}\right\rangle\right\rangle_{1}\left\langle\left\langle\gamma_{\alpha} \gamma_{\beta} \gamma^{\beta} \gamma_{\mu} \gamma^{\mu}\right\rangle\right\rangle_{0}+a_{20}\left\langle\left\langle\gamma^{\alpha}\right\rangle\right\rangle_{1}\left\langle\left\langle\gamma_{\alpha} \mathcal{W} \gamma^{\beta} \gamma_{\beta} \gamma^{\mu} \gamma_{\mu}\right\rangle\right\rangle_{0} \\
& +a_{21}\left\langle\left\langle\mathcal{W} \gamma^{\alpha} \gamma^{\beta}\right\rangle\right\rangle_{1}\left\langle\left\langle\gamma_{\alpha} \gamma_{\beta} \gamma^{\mu} \gamma_{\mu}\right\rangle_{0}+a_{22}\left\langle\left\langle\gamma^{\alpha} \gamma^{\beta}\right\rangle\right\rangle_{1}\left\langle\left\langle\gamma_{\alpha} \gamma_{\beta} \mathcal{W} \gamma^{\mu} \gamma_{\mu}\right\rangle\right\rangle_{0}\right. \\
& +a_{23}\left\langle\left\langle\mathcal{W} \gamma^{\alpha} \gamma_{\alpha} \gamma^{\beta} \gamma_{\beta} \gamma^{\mu} \gamma_{\mu}\right\rangle\right\rangle_{0}+a_{24}\left\langle\left\langle\left\{\mathcal{W} \circ \gamma^{\alpha}\right\}\right\rangle_{1}\left\langle\left\langle\gamma_{\alpha} \gamma^{\beta} \gamma_{\beta}\right\rangle\right\rangle_{1}\right. \\
& +a_{25}\left\langle\left\langle\mathcal{W} \gamma^{\alpha} \gamma_{\alpha}\left\{\gamma^{\beta} \circ \gamma_{\beta}\right\}\right\rangle\right\rangle_{1}+a_{26}\left\langle\left\langle\gamma^{\alpha} \gamma_{\alpha} \gamma^{\beta}\right\rangle\right\rangle_{1}\left\langle\left\langle\gamma_{\beta} \mathcal{W} \gamma^{\mu} \gamma_{\mu}\right\rangle_{0}\right. \\
& +a_{27}\left\langle\left\langle\gamma^{\alpha} \gamma_{\alpha} \gamma^{\beta}\left\{\gamma_{\beta} \circ \mathcal{W}\right\}\right\rangle\right\rangle_{1}+a_{28}\left\langle\left\langle T\left(\gamma_{\alpha}\right)\left\{\mathcal{W} \circ \gamma^{\alpha}\right\}\right\rangle\right\rangle_{2} \\
& +a_{29}\left\langle\left\langle\mathcal{W} \gamma_{\alpha} \gamma_{\beta} \gamma_{\mu}\right\rangle\right\rangle_{1}\left\langle\left\langle\gamma^{\alpha} \gamma^{\beta} \gamma^{\mu}\right\rangle\right\rangle_{0}+a_{30}\left\langle\left\langle\gamma_{\alpha} \gamma_{\beta} \gamma_{\mu}\right\rangle\right\rangle_{1}\left\langle\left\langle\mathcal{W} \gamma^{\alpha} \gamma^{\beta} \gamma^{\mu}\right\rangle\right\rangle_{0}
\end{aligned}
$$

where $\mathcal{W}$ is any vector field on the big phase space.

Using the genus-2 equation discovered by Belurousski-Pandharipande $[\mathrm{BP}$, we can write

$$
\left\langle\left\langle T(\mathcal{W})\left\{\gamma_{\alpha} \circ \gamma^{\alpha}\right\}\right\rangle_{2}\right.
$$

as a linear combination of other terms on the right hand side of equation (2) (cf. [L1, Equation (21)]). Therefore, we can set

$$
a_{1}=0 .
$$

Note that equation (2) holds for any compact symplectic manifold. However, we shall see that the Gromov-Witten invariants of a point and of $\mathbb{C} P^{1}$ already completely determine the coefficients $a_{2}, \ldots, a_{30}$.

\subsection{Relations obtained from the Gromov-Witten invariants of a point}

When the target manifold is a point, all stable maps must necessarily have degree 0 . Hence, we will omit any reference to the degrees of these Gromov-Witten invariants. In fact, the moduli space of stable maps into a point is isomorphic to the moduli space of stable curves. 
Since the cohomology space of a point is one dimensional, coordinates on the big phase space are simply denoted by $t_{0}, t_{1}, t_{2}, \cdots$. We also identify vector fields $\frac{\partial}{\partial t_{m}}$ with $\tau_{m}$ on the big phase space.

Gromov-Witten invariants of a point obey the string equation

$$
\left\langle\tau_{0} \tau_{n_{1}} \cdots \tau_{n_{k}}\right\rangle_{g}=\sum_{j=1}^{k}\left\langle\tau_{n_{1}} \cdots \tau_{n_{j}-1} \cdots \tau_{n_{k}}\right\rangle_{g, d}+\delta_{g, 0} \delta_{k, 2} \delta_{n_{1}, 0} \delta_{n_{2}, 0}
$$

and the dilaton equation

$$
\left\langle\tau_{1} \tau_{n_{1}} \cdots \tau_{n_{k}}\right\rangle_{g}=(2 g-2+k)\left\langle\tau_{n_{1}} \cdots \tau_{n_{k}}\right\rangle_{g}+\frac{1}{24} \delta_{g, 1} \delta_{k, 0} .
$$

We can use these two equations to compute Gromov-Witten invariants involving only $\tau_{0}$ and $\tau_{1}$. More complicated Gromov-Witten invariants of a point can be computed using the Virasoro constraints, or equivalently the KdV hierarchy which were conjectured by Witten and proven by Kontsevich (cf. [W] and [K]). To determine the coefficients $a_{2}, \ldots, a_{30}$ in equation (11), we will only need the genus-2 invariants

$$
\left\langle\tau_{4}\right\rangle_{2}=\frac{1}{1152}, \quad\left\langle\tau_{2} \tau_{3}\right\rangle_{2}=\frac{29}{5760}, \quad\left\langle\tau_{2} \tau_{2} \tau_{2}\right\rangle_{2}=\frac{7}{240}
$$

and the genus-3 invariants

$$
\begin{array}{lll}
\left\langle\tau_{7}\right\rangle_{3}=\frac{1}{82944}, & \left\langle\tau_{2} \tau_{6}\right\rangle_{3}=\frac{77}{414720}, & \left\langle\tau_{3} \tau_{5}\right\rangle_{3}=\frac{503}{1451520}, \\
\left\langle\tau_{4} \tau_{4}\right\rangle_{3}=\frac{607}{1451520}, & \left\langle\tau_{2} \tau_{2} \tau_{5}\right\rangle_{3}=\frac{17}{5760}, & \left\langle\tau_{2} \tau_{3} \tau_{4}\right\rangle_{3}=\frac{1121}{241920}, \\
\left\langle\tau_{3} \tau_{3} \tau_{3}\right\rangle_{3}=\frac{583}{96768}, & \left\langle\tau_{2} \tau_{2} \tau_{2} \tau_{4}\right\rangle_{3}=\frac{53}{1152}, & \left\langle\tau_{2} \tau_{2} \tau_{3} \tau_{3}\right\rangle_{3}=\frac{205}{3456}, \\
\left\langle\tau_{2} \tau_{2} \tau_{2} \tau_{2} \tau_{3}\right\rangle_{3}=\frac{193}{288} . &
\end{array}
$$

All other invariants needed to determine the coefficients $a_{2}, \ldots, a_{30}$ can be computed from the string and dilaton equations.

We will compute derivatives of $\Phi\left(\tau_{m}\right)$ restricted to the origin $t=0$ of the big phase space. By equation (2), these values must all be equal to zero. We thus obtain some linear relations between the coefficients $a_{2}, \ldots, a_{30}$ in equation (II).

From $\left.\Phi\left(\tau_{4}\right)\right|_{t=0}=0$, we obtain

$$
0=-\frac{1}{82944}+\frac{a_{2}}{384}+a_{23}+\frac{a_{25}}{24}+\frac{a_{29}}{24} .
$$

From $\left.\tau_{5} \Phi\left(\tau_{0}\right)\right|_{t=0}=0$, we obtain

$$
0=-\frac{503}{1451520}+\frac{a_{2}}{288}+\frac{a_{5}}{1152}+a_{23}+\frac{a_{25}}{24}+\frac{a_{27}}{24}+\frac{a_{28}}{288}+\frac{a_{29}}{24} .
$$


From $\left.\tau_{4} \Phi\left(\tau_{1}\right)\right|_{t=0}=0$, we obtain

$$
0=-\frac{607}{1451520}+\frac{a_{2}}{96}+\frac{a_{3}}{384}+5 a_{23}+\frac{a_{25}}{6}+\frac{a_{26}}{24}+\frac{a_{29}}{6}+\frac{a_{30}}{24} .
$$

From $\left.\tau_{3} \Phi\left(\tau_{2}\right)\right|_{t=0}=0$, we obtain

$$
0=-\frac{503}{1451520}+\frac{29 a_{2}}{1440}+\frac{a_{6}}{576}+\frac{a_{18}}{576}+\frac{a_{19}}{24}+\frac{a_{22}}{24}+10 a_{23}+\frac{7 a_{25}}{24}+\frac{7 a_{29}}{24} .
$$

From $\left.\tau_{2} \Phi\left(\tau_{3}\right)\right|_{t=0}=0$, we obtain

$$
0=-\frac{77}{414720}+\frac{29 a_{2}}{1440}+\frac{a_{7}}{576}+\frac{a_{16}}{576}+\frac{a_{20}}{24}+\frac{a_{21}}{24}+10 a_{23}+\frac{7 a_{25}}{24}+\frac{7 a_{29}}{24} .
$$

From $\left.\tau_{3} \tau_{3} \Phi\left(\tau_{0}\right)\right|_{t=0}=0$, we obtain

$$
\begin{aligned}
0= & -\frac{583}{96768}+\frac{29 a_{2}}{576}+\frac{29 a_{5}}{2880}+\frac{a_{6}}{288}+\frac{a_{11}}{288}+\frac{a_{18}}{288}+\frac{a_{19}}{12}+\frac{a_{22}}{12} \\
& +20 a_{23}+\frac{7 a_{25}}{12}+\frac{7 a_{27}}{12}+\frac{29 a_{28}}{576}+\frac{7 a_{29}}{12} .
\end{aligned}
$$

From $\left.\tau_{2} \tau_{4} \Phi\left(\tau_{0}\right)\right|_{t=0}=0$, we obtain

$$
\begin{aligned}
0= & -\frac{1121}{241920}+\frac{11 a_{2}}{288}+\frac{a_{3}}{384}+\frac{a_{4}}{9216}+\frac{11 a_{5}}{1440}+\frac{a_{7}}{576}+\frac{a_{15}}{576}+\frac{a_{16}}{576}+\frac{a_{20}}{24} \\
& +\frac{a_{21}}{24}+15 a_{23}+\frac{a_{24}}{576}+\frac{11 a_{25}}{24}+\frac{a_{26}}{24}+\frac{11 a_{27}}{24}+\frac{11 a_{28}}{288}+\frac{11 a_{29}}{24}+\frac{a_{30}}{24} .
\end{aligned}
$$

From $\left.\tau_{2} \tau_{3} \Phi\left(\tau_{1}\right)\right|_{t=0}=0$, we obtain

$$
\begin{aligned}
0= & -\frac{1121}{241920}+\frac{29 a_{2}}{288}+\frac{29 a_{3}}{1440}+\frac{a_{6}}{288}+\frac{a_{7}}{192}+\frac{a_{8}}{576}+\frac{a_{16}}{192}+\frac{a_{17}}{576}+\frac{a_{18}}{288} \\
& +\frac{a_{19}}{12}+\frac{a_{20}}{6}+\frac{a_{21}}{8}+\frac{a_{22}}{8}+60 a_{23}+\frac{35 a_{25}}{24}+\frac{7 a_{26}}{24}+\frac{35 a_{29}}{24}+\frac{7 a_{30}}{24} .
\end{aligned}
$$

From $\left.\tau_{2} \tau_{2} \Phi\left(\tau_{2}\right)\right|_{t=0}=0$, we obtain

$$
\begin{aligned}
0= & -\frac{17}{5760}+\frac{7 a_{2}}{48}+\frac{a_{6}}{144}+\frac{a_{7}}{72}+\frac{a_{9}}{288}+\frac{a_{10}}{288}+\frac{a_{13}}{6912}+\frac{a_{16}}{72} \\
& +\frac{a_{18}}{144}+\frac{a_{19}}{4}+\frac{a_{20}}{2}+\frac{a_{21}}{3}+\frac{a_{22}}{6}+90 a_{23}+2 a_{25}+2 a_{29} .
\end{aligned}
$$

From $\left.\tau_{2} \tau_{2} \tau_{3} \Phi\left(\tau_{0}\right)\right|_{t=0}=0$, we obtain

$$
\begin{aligned}
0= & -\frac{205}{3456}+\frac{5 a_{2}}{12}+\frac{29 a_{3}}{720}+\frac{29 a_{4}}{17280}+\frac{5 a_{5}}{72}+\frac{a_{6}}{72}+\frac{7 a_{7}}{288}+\frac{a_{8}}{288}+\frac{a_{9}}{288}+\frac{a_{10}}{288} \\
& +\frac{a_{11}}{72}+\frac{a_{12}}{6912}+\frac{a_{13}}{6912}+\frac{7 a_{15}}{288}+\frac{7 a_{16}}{288}+\frac{a_{17}}{288}+\frac{a_{18}}{72}+\frac{5 a_{19}}{12}+\frac{5 a_{20}}{6}+\frac{7 a_{21}}{12}+\frac{5 a_{22}}{12} \\
& +210 a_{23}+\frac{7 a_{24}}{288}+\frac{59 a_{25}}{12}+\frac{7 a_{26}}{12}+\frac{59 a_{27}}{12}+\frac{5 a_{28}}{12}+\frac{59 a_{29}}{12}+\frac{7 a_{30}}{12} .
\end{aligned}
$$


From $\left.\tau_{2} \tau_{2} \tau_{2} \Phi\left(\tau_{1}\right)\right|_{t=0}=0$, we obtain

$$
\begin{aligned}
0= & -\frac{53}{1152}+\frac{7 a_{2}}{8}+\frac{7 a_{3}}{48}+\frac{a_{6}}{24}+\frac{a_{7}}{12}+\frac{a_{8}}{48}+\frac{a_{9}}{48}+\frac{a_{10}}{32}+\frac{a_{13}}{1152}+\frac{a_{14}}{2304}+\frac{a_{16}}{12} \\
& +\frac{a_{17}}{48}+\frac{a_{18}}{24}+\frac{3 a_{19}}{2}+\frac{15 a_{20}}{4}+2 a_{21}+\frac{3 a_{22}}{2}+630 a_{23} \\
& +12 a_{25}+2 a_{26}+12 a_{29}+2 a_{30} .
\end{aligned}
$$

From $\left.\tau_{2} \tau_{2} \tau_{2} \tau_{2} \Phi\left(\tau_{0}\right)\right|_{t=0}=0$, we obtain

$$
\begin{aligned}
0= & -\frac{193}{288}+\frac{49 a_{2}}{12}+\frac{7 a_{3}}{12}+\frac{7 a_{4}}{288}+\frac{7 a_{5}}{12}+\frac{a_{6}}{6}+\frac{a_{7}}{3}+\frac{a_{8}}{12}+\frac{a_{9}}{12}+\frac{a_{10}}{8}+\frac{a_{11}}{6} \\
& +\frac{a_{12}}{288}+\frac{a_{13}}{288}+\frac{a_{14}}{576}+\frac{a_{15}}{3}+\frac{a_{16}}{3}+\frac{a_{17}}{12}+\frac{a_{18}}{6}+6 a_{19}+15 a_{20}+8 a_{21}+6 a_{22} \\
& +2520 a_{23}+\frac{a_{24}}{3}+48 a_{25}+8 a_{26}+48 a_{27}+\frac{49 a_{28}}{12}+48 a_{29}+8 a_{30} .
\end{aligned}
$$

\subsection{Relations obtained from the Gromov-Witten invariants of $\mathbb{C} P^{1}$}

When the target manifold is $\mathbb{C} P^{1}$, the degrees of the stable maps are indexed by $H_{2}\left(\mathbb{C} P^{1} ; \mathbb{Z}\right) \cong$ $\mathbb{Z}$. The degree $d$ part of any equation for generating functions of Gromov-Witten invariants is the coefficient of $q^{d}$ in the Novikov ring. We choose the basis $\left\{\gamma_{0}, \gamma_{1}\right\}$ for $H^{*}\left(\mathbb{C} P^{1} ; \mathbb{C}\right)$ with $\gamma_{0} \in H^{0}\left(\mathbb{C} P^{1} ; \mathbb{C}\right)$ being the identity of the ordinary cohomology ring and $\gamma_{1} \in H^{2}\left(\mathbb{C} P^{1} ; \mathbb{C}\right)$ the Poincare dual to a point. Coordinates on the big phase space are denoted by $\left\{t_{n}^{0}, t_{n}^{1} \mid n \in \mathbb{Z}_{+}\right\}$. We identify vector fields $\frac{\partial}{\partial t_{n}^{0}}$ and $\frac{\partial}{\partial t_{n}^{1}}$ with $\tau_{n, 0}$ and $\tau_{n, 1}$ respectively. We also define $\tau_{n, \alpha}=0$ if $n<0$.

The Gromov-Witten invariants of $\mathbb{C} P^{1}$ obey three basic equations: the string equation

$$
\begin{aligned}
\left\langle\tau_{0,0} \tau_{n_{1}, \alpha_{1}} \cdots \tau_{n_{k}, \alpha_{k}}\right\rangle_{g, d}= & \sum_{j=1}^{k}\left\langle\tau_{n_{1}, \alpha_{1}} \cdots \tau_{n_{j}-1, \alpha_{j}} \cdots \tau_{n_{k}, \alpha_{k}}\right\rangle_{g, d} \\
& +\delta_{g, 0} \delta_{d, 0} \delta_{k, 2} \delta_{n_{1}, 0} \delta_{n_{2}, 0} \delta_{\alpha_{1}+\alpha_{2}, 1}
\end{aligned}
$$

the dilaton equation

$$
\left\langle\tau_{1,0} \tau_{n_{1}, \alpha_{1}} \cdots \tau_{n_{k}, \alpha_{k}}\right\rangle_{g, d}=(2 g-2+k)\left\langle\tau_{n_{1}, \alpha_{1}} \cdots \tau_{n_{k}, \alpha_{k}}\right\rangle_{g, d}+\frac{1}{12} \delta_{g, 1} \delta_{k, 0} \delta_{d, 0}
$$

and the divisor equation

$$
\begin{aligned}
\left\langle\tau_{0,1} \tau_{n_{1}, \alpha_{1}} \cdots \tau_{n_{k}, \alpha_{k}}\right\rangle_{g, d}= & d\left\langle\tau_{n_{1}, \alpha_{1}} \cdots \tau_{n_{k}, \alpha_{k}}\right\rangle_{g, d} \\
& +\sum_{j=1}^{k} \delta_{\alpha_{j}, 0}\left\langle\tau_{n_{1}, \alpha_{1}} \cdots \tau_{n_{j}-1,1} \cdots \tau_{n_{k}, \alpha_{k}}\right\rangle_{g, d} \\
& +\delta_{g, 0} \delta_{d, 0} \delta_{k, 2} \delta_{n_{1}, 0} \delta_{n_{2}, 0} \delta_{\alpha_{1}, 0} \delta_{\alpha_{2}, 0}-\frac{1}{24} \delta_{g, 1} \delta_{d, 0} \delta_{k, 0}
\end{aligned}
$$


We can use these three equations to compute Gromov-Witten invariants involving only $\tau_{0,0}, \tau_{1,0}$, and $\tau_{0,1}$. More complicated Gromov-Witten invariants for $\mathbb{C} P^{1}$ can be computed using the Virasoro constraints which was conjectured in [EHX] and proven in [Gi]. A computer program for computing such invariants based on the Virasoro constraints was written by Andreas Gathmann (cf. Ga ). To determine $a_{2}, \cdots a_{30}$ in equation (1), we only need a small number of such invariants. In Appendix $\mathrm{A}$ we will list all of the necessary invariants which are obtained from Gathmann's program.

To obtain more relations on $a_{2}, \cdots a_{30}$ in equation (11), we will compute derivatives of $\Phi\left(\tau_{m, \alpha}\right)$ at the origin of the big phase space $t=0$. From degree 0 part of $\left.\Phi\left(\tau_{1,1}\right)\right|_{t=0}=0$, we obtain

$$
0=\frac{31}{967680}-\frac{a_{13}}{13824}-\frac{a_{14}}{13824} .
$$

From degree 0 part of $\left.\Phi\left(\tau_{2,0}\right)\right|_{t=0}=0$, we obtain

$$
0=-\frac{41}{290304}+\frac{7 a_{2}}{960}+\frac{a_{7}}{288}+\frac{a_{10}}{288}+\frac{a_{13}}{6912}+\frac{a_{16}}{288} .
$$

From degree 1 part of $\left.\Phi\left(\tau_{3,1}\right)\right|_{t=0}=0$, we obtain

$$
\begin{aligned}
0= & -\frac{1}{322560}-\frac{7 a_{2}}{2880}-\frac{a_{7}}{288}+\frac{a_{10}}{288}+\frac{a_{13}}{13824} \\
& -\frac{a_{14}}{13824}-\frac{a_{16}}{288}-\frac{a_{20}}{6}+8 a_{23}+\frac{a_{25}}{6}+\frac{a_{29}}{12} .
\end{aligned}
$$

From degree 0 part of $\left.\tau_{2,1} \Phi\left(\tau_{0,0}\right)\right|_{t=0}=0$, we obtain

$$
0=\frac{31}{96768}-\frac{7 a_{4}}{46080}-\frac{a_{12}}{13824}-\frac{a_{13}}{13824}-\frac{a_{14}}{13824} .
$$

From degree 0 part of $\left.\tau_{3,0} \Phi\left(\tau_{0,0}\right)\right|_{t=0}=0$, we obtain

$$
\begin{aligned}
0= & -\frac{1501}{725760}+\frac{7 a_{2}}{720}+\frac{a_{4}}{1920}+\frac{7 a_{5}}{2880}+\frac{a_{7}}{288}+\frac{a_{10}}{288}+\frac{a_{12}}{6912} \\
& +\frac{a_{13}}{6912}+\frac{a_{15}}{288}+\frac{a_{16}}{288}+\frac{a_{24}}{288}+\frac{7 a_{28}}{720} .
\end{aligned}
$$

From degree 0 part of $\left.\tau_{2,0} \Phi\left(\tau_{1,0}\right)\right|_{t=0}=0$, we obtain

$$
\begin{aligned}
0= & -\frac{2329}{1451520}+\frac{7 a_{2}}{240}+\frac{7 a_{3}}{960}+\frac{a_{6}}{288}+\frac{a_{7}}{96}+\frac{a_{8}}{288}+\frac{a_{9}}{288}+\frac{a_{10}}{96}+\frac{a_{13}}{1728} \\
& +\frac{a_{14}}{2304}+\frac{a_{16}}{96}+\frac{a_{17}}{288}+\frac{a_{18}}{288} .
\end{aligned}
$$

From degree 1 part of $\left.\tau_{3,1} \Phi\left(\tau_{0,1}\right)\right|_{t=0}=0$, we obtain

$$
\begin{aligned}
0= & -\frac{31}{96768}+\frac{7 a_{4}}{138240}+\frac{7 a_{5}}{2880}-\frac{a_{7}}{288}+\frac{a_{10}}{288}+\frac{a_{12}}{13824}+\frac{a_{13}}{13824}-\frac{a_{14}}{13824} \\
& -\frac{a_{16}}{288}-\frac{a_{20}}{6}+8 a_{23}-\frac{a_{24}}{288}+\frac{a_{25}}{6}+\frac{a_{27}}{12}+\frac{7 a_{28}}{1440}+\frac{a_{29}}{8}-\frac{a_{30}}{24} .
\end{aligned}
$$


From degree 1 part of $\left.\tau_{4,0} \Phi\left(\tau_{0,1}\right)\right|_{t=0}=0$, we obtain

$$
\begin{aligned}
0= & \frac{277}{207360}+\frac{a_{2}}{360}-\frac{13 a_{4}}{46080}-\frac{a_{5}}{120}-\frac{a_{7}}{144}+\frac{a_{12}}{13824}+\frac{a_{13}}{13824}+\frac{a_{14}}{13824}-\frac{a_{15}}{144} \\
& -\frac{a_{16}}{144}-\frac{a_{20}}{6}+16 a_{23}-\frac{a_{24}}{144}+\frac{a_{25}}{2}+\frac{a_{27}}{6}-\frac{11 a_{28}}{720}+\frac{a_{29}}{3}+\frac{a_{30}}{12} .
\end{aligned}
$$

From degree 1 part of $\left.\tau_{3,1} \Phi\left(\tau_{1,0}\right)\right|_{t=0}=0$, we obtain

$$
\begin{aligned}
0= & \frac{41}{193536}-\frac{7 a_{2}}{720}-\frac{7 a_{3}}{2880}-\frac{a_{6}}{288}-\frac{a_{7}}{96}-\frac{a_{8}}{288}+\frac{a_{9}}{288}+\frac{a_{10}}{96}+\frac{a_{13}}{4608}+\frac{a_{14}}{13824} \\
& -\frac{a_{16}}{96}-\frac{a_{17}}{288}-\frac{a_{18}}{288}-\frac{a_{19}}{6}-\frac{2 a_{20}}{3}+40 a_{23}+\frac{2 a_{25}}{3}+\frac{a_{26}}{6}+\frac{a_{29}}{3}+\frac{a_{30}}{12} .
\end{aligned}
$$

From degree 1 part of $\left.\tau_{2,1} \Phi\left(\tau_{1,1}\right)\right|_{t=0}=0$, we obtain

$$
\begin{aligned}
0= & -\frac{1}{46080}+\frac{7 a_{3}}{960}-\frac{7 a_{4}}{46080}-\frac{a_{6}}{288}-\frac{a_{7}}{288}+\frac{a_{8}}{288}+\frac{a_{9}}{288}+\frac{a_{10}}{96}-\frac{a_{12}}{13824} \\
& +\frac{a_{13}}{6912}-\frac{a_{16}}{288}-\frac{a_{19}}{6}-\frac{a_{20}}{2}-\frac{a_{22}}{12}+24 a_{23}+\frac{a_{25}}{6}+\frac{a_{29}}{8}+\frac{a_{30}}{24} .
\end{aligned}
$$

From degree 1 part of $\left.\tau_{3,0} \Phi\left(\tau_{1,1}\right)\right|_{t=0}=0$, we obtain

$$
\begin{aligned}
0= & -\frac{83}{193536}-\frac{7 a_{2}}{720}-\frac{a_{3}}{40}+\frac{a_{4}}{1920}+\frac{7 a_{5}}{2880}-\frac{a_{6}}{144}-\frac{5 a_{7}}{288}-\frac{a_{8}}{144}+\frac{a_{10}}{288}+\frac{a_{12}}{6912} \\
& +\frac{7 a_{13}}{13824}+\frac{5 a_{14}}{13824}+\frac{a_{15}}{288}-\frac{5 a_{16}}{288}-\frac{a_{17}}{144}-\frac{a_{18}}{144}-\frac{a_{19}}{6}-\frac{2 a_{20}}{3}+\frac{a_{22}}{6}+56 a_{23} \\
& +\frac{a_{24}}{288}+a_{25}-\frac{a_{26}}{6}+\frac{7 a_{28}}{720}+\frac{7 a_{29}}{12} .
\end{aligned}
$$

From degree 1 part of $\left.\tau_{2,1} \Phi\left(\tau_{2,0}\right)\right|_{t=0}=0$, we obtain

$$
\begin{aligned}
0= & -\frac{19}{138240}+\frac{a_{2}}{320}+\frac{7 a_{4}}{46080}+\frac{a_{6}}{144}-\frac{5 a_{7}}{288}-\frac{a_{9}}{144}+\frac{a_{10}}{288}+\frac{a_{12}}{13824} \\
& +\frac{a_{13}}{6912}-\frac{5 a_{16}}{288}+\frac{a_{19}}{3}-a_{20}-\frac{a_{21}}{6}+72 a_{23}+a_{25}+\frac{a_{29}}{2} .
\end{aligned}
$$

From degree 1 part of $\left.\tau_{1,1} \Phi\left(\tau_{2,1}\right)\right|_{t=0}=0$, we obtain

$$
\begin{aligned}
0= & \frac{7 a_{2}}{960}-\frac{a_{7}}{288}+\frac{5 a_{10}}{288}+\frac{a_{13}}{3456}-\frac{a_{14}}{2304}-\frac{a_{16}}{288} \\
& -\frac{2 a_{20}}{3}-\frac{a_{21}}{12}+24 a_{23}+\frac{a_{25}}{6}+\frac{a_{29}}{6} .
\end{aligned}
$$

From degree 1 part of $\left.\tau_{2,0} \Phi\left(\tau_{2,1}\right)\right|_{t=0}=0$, we obtain

$$
\begin{aligned}
0= & -\frac{1}{15360}-\frac{a_{2}}{240}+\frac{7 a_{3}}{960}-\frac{7 a_{4}}{46080}-\frac{a_{6}}{288}-\frac{a_{7}}{96}+\frac{a_{8}}{288}-\frac{a_{9}}{288}-\frac{a_{10}}{288}-\frac{a_{12}}{13824} \\
& +\frac{a_{14}}{2304}-\frac{5 a_{16}}{288}+\frac{a_{17}}{288}-\frac{a_{18}}{288}-\frac{2 a_{20}}{3}-\frac{a_{22}}{6}+72 a_{23}+a_{25}+\frac{a_{29}}{2} .
\end{aligned}
$$


From degree 1 part of $\left.\tau_{1,1} \Phi\left(\tau_{3,0}\right)\right|_{t=0}=0$, we obtain

$$
\begin{aligned}
0= & -\frac{1}{46080}-\frac{107 a_{2}}{2880}-\frac{a_{7}}{32}+\frac{a_{10}}{288}+\frac{7 a_{13}}{13824}+\frac{5 a_{14}}{13824}-\frac{a_{16}}{32} \\
& -\frac{5 a_{20}}{6}+\frac{a_{21}}{6}+56 a_{23}+\frac{5 a_{25}}{6}+\frac{7 a_{29}}{12} .
\end{aligned}
$$

From degree 1 part of $\left.\tau_{1,1} \tau_{2,1} \Phi\left(\tau_{1,0}\right)\right|_{t=0}=0$, we obtain

$$
\begin{aligned}
0= & -\frac{1}{9216}+\frac{7 a_{2}}{240}+\frac{7 a_{3}}{480}-\frac{7 a_{4}}{46080}-\frac{a_{6}}{144}-\frac{a_{7}}{72}+\frac{a_{9}}{48}+\frac{a_{10}}{16}-\frac{a_{12}}{13824} \\
& +\frac{a_{13}}{1152}-\frac{a_{16}}{72}-\frac{a_{17}}{288}-\frac{a_{18}}{288}-\frac{5 a_{19}}{6}-\frac{19 a_{20}}{6}-\frac{a_{21}}{4}-\frac{a_{22}}{4}+144 a_{23} \\
& +\frac{5 a_{25}}{6}+\frac{a_{26}}{6}+\frac{19 a_{29}}{24}+\frac{5 a_{30}}{24} .
\end{aligned}
$$

From degree 1 part of $\left.\tau_{2,0} \tau_{2,0} \Phi\left(\tau_{1,1}\right)\right|_{t=0}=0$, we obtain

$$
\begin{aligned}
0= & -\frac{661}{161280}-\frac{a_{2}}{96}-\frac{179 a_{3}}{1440}+\frac{263 a_{4}}{69120}+\frac{7 a_{5}}{480}-\frac{7 a_{6}}{144}-\frac{7 a_{7}}{144}-\frac{5 a_{8}}{144}-\frac{5 a_{9}}{144} \\
& -\frac{5 a_{10}}{144}+\frac{a_{11}}{144}+\frac{a_{12}}{768}-\frac{a_{13}}{1152}+\frac{a_{15}}{48}-\frac{13 a_{16}}{144}-\frac{5 a_{17}}{144}-\frac{7 a_{18}}{144}-a_{19}-\frac{5 a_{20}}{3} \\
& -\frac{a_{22}}{3}+528 a_{23}+\frac{a_{24}}{48}+\frac{19 a_{25}}{3}-a_{26}+\frac{7 a_{28}}{96}+\frac{7 a_{29}}{2}-\frac{a_{30}}{6} .
\end{aligned}
$$

Remark: We have also checked more than 18000 other combinations of derivatives of $\Phi$ for the $\mathbb{C} P^{1}$ case using a Mathematica program, but the relations obtained are just linear combinations of the relations (41) to (32). This explicitly verifies that these relations are indeed consistent.

\subsection{Proof of Theorem 0.1}

It is straightforward to solve $a_{2}, \ldots, a_{30}$ from equations (41) to (32). The answers are

$$
\begin{array}{llll}
a_{2}=-\frac{1}{252}, & a_{3}=\frac{13}{168}, & a_{4}=\frac{41}{21}, & a_{5}=-\frac{13}{168}, \\
a_{6}=\frac{1}{280}, & a_{7}=-\frac{23}{5040}, & a_{8}=-\frac{47}{5040}, & a_{9}=-\frac{5}{1008}, \\
a_{10}=\frac{23}{504}, & a_{11}=\frac{11}{140}, & a_{12}=-\frac{4}{35}, & a_{13}=\frac{2}{105}, \\
a_{14}=\frac{89}{210}, & a_{15}=-\frac{1}{210}, & a_{16}=\frac{1}{140}, & a_{17}=\frac{23}{140}, \\
a_{18}=-\frac{3}{140}, & a_{19}=-\frac{1}{4480}, & a_{20}=\frac{13}{8064}, & a_{21}=-\frac{1}{2240}, \\
a_{22}=\frac{41}{6720}, & a_{23}=\frac{1}{53760}, & a_{24}=-\frac{1}{210}, & a_{25}=-\frac{1}{5760}, \\
a_{26}=-\frac{1}{2688}, & a_{27}=-\frac{1}{5040}, & a_{28}=\frac{5}{42}, & a_{29}=\frac{1}{3780}, \\
a_{30}=\frac{1}{252} . & &
\end{array}
$$

Together with equations (3) and (2), this proves equation (11).

We make the following observations about topological recursion relations of genus less than or equal to 3 : 
1. $T(\mathcal{W})$ does not appear in the lower genus terms of the genus- $g$ topological recursion relations for $T^{g}(\mathcal{W})$ when $g=1,2,3$.

2. With the exception of $a_{25}$, all of the denominators in the coefficients of the genus-3 topological recursion relation (11) have a factor 7 . We also note that the genus2 topological recursion relations (i.e. Mumford's equation), have denominators in its coefficients of lower genus terms contain a factor of 10 , while for the genus-1 topological recursion relation, the corresponding factor is 24 .

3. For $g=1,2,3$, the coefficients of the terms consisting of purely genus- 0 functions in the genus- $g$ topological recursion relations are

$$
\frac{1}{(2 g+1) ! ! 8^{g}} \text {. }
$$

We conjecture that this should also hold for all genera.

\section{A new relation in tautological ring of $\overline{\mathcal{M}}_{3,1}$}

Note that equation (2) is a direct translation of a relation in the tautological ring of $\overline{\mathcal{M}}_{3,1}$ with undetermined coefficients $a_{1}, \cdots a_{30}$. Those coefficients were determined during the proof of topological recursion relation (11). Therefore we have also obtained a proof to the following theorem:

Theorem 3.1 In the tautological ring of $\overline{\mathcal{M}}_{3,1}$, the following relation holds

$$
\begin{aligned}
& \psi_{1}^{3}=-\frac{1}{126} \quad \stackrel{1}{2}+\frac{13}{84} \quad 2 \longleftarrow+\frac{5}{42} \\
& +\frac{41}{21} \\
& +\frac{1}{140} \stackrel{1}{1}-\frac{23}{2520} \\
& -\frac{47}{2520} \text { (1) }-\frac{1}{105} \text { (1) } \\
& +\frac{11}{70} \text { (1) } \\
& -\frac{1}{105} \text { (1) }
\end{aligned}
$$




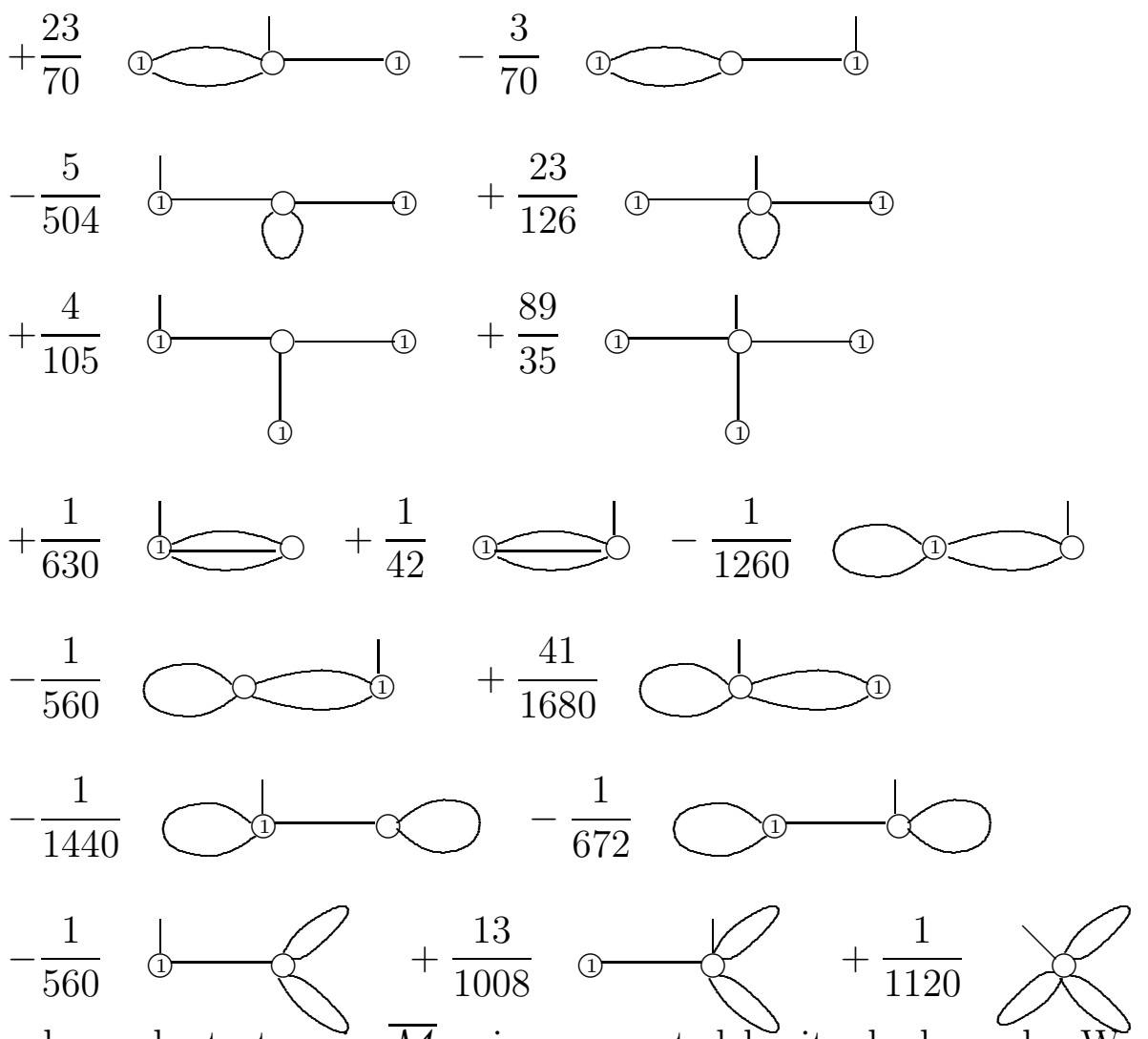

In this formula, each stratum in $\overline{\mathcal{M}}_{g, n}$ is represented by its dual graph. We adopt the conventions of [Ge2] for dual graphs with a slight modification. We denote vertices of genus 0 by a hollow circle $\bigcirc$, and vertices of genus $g \geq 1$ by (9). A vertex with an incident arrowhead denotes the $\psi$ class associated to the marked point (which is at a node) on the irreducible component associated to that vertex.

Note that when translating relations in the tautological ring of $\overline{\mathcal{M}}_{g, n}$ to GromovWitten invariants, we need to divide the coefficient of each stratum by the number of elements in the automorphism group of the corresponding dual graph. This explains the difference between the coefficients in this formula and those in equation (10).

\section{Application to Higher Spin Curves}

We briefly review the moduli space of $r$-spin curves and $r$-spin theory. For details, we refer the reader to [JKV]. Let $r \geq 2$ be an integer. For each $r, r$-spin theory is a cohomological field theory, in the sense of Kontsevich-Manin, just as is the Gromov-Witten theory of a compact, symplectic manifold $M$. Consequently, the correlation functions in this theory satisfy the same universal equations as does Gromov-Witten theory. In particular, they satisfy the topological recursion relations, the string, and dilaton equations. However, there is no analog of the divisor equation but we will not need this.

The role of the moduli space of stable maps is replaced by the moduli space of $r$ spin curves $\overline{\mathcal{M}}_{g, n}^{1 / r}$. Let $[n]:=\{0, \ldots, n\}$ for any nonnegative integer $n$ then we have 
the disjoint union $\overline{\mathcal{M}}_{g, n}^{1 / r}:=\bigsqcup_{\boldsymbol{\alpha} \in[r-2]^{n}} \overline{\mathcal{M}}_{g, n}^{1 / r}(\boldsymbol{\alpha})$. While the definition of an $r$-spin curve is rather involved, over a smooth stable curve $\left(C ; p_{1}, \ldots, p_{n}\right)$ of genus $g$ with $n$ marked points when $r$ is prime, a point in $\overline{\mathcal{M}}_{g, n}^{1 / r}(\boldsymbol{\alpha})$ consists of a line bundle $\mathcal{L} \rightarrow C$ together with an isomorphism $\mathcal{L}^{\otimes r} \rightarrow \omega\left(-\sum_{i=1}^{n} \alpha_{i} p_{i}\right)$ where $\boldsymbol{\alpha}=\left(\alpha_{1}, \ldots, \alpha_{n}\right)$ and $\omega$ is the canonical bundle of $C$. For degree reasons, such a line bundle exists only if $\left(2-2 g+\sum_{i=1}^{n} \alpha_{i}\right) / r$ is an integer. When $r$ is not prime then one should introduce all $d$-th roots for all $d$ which divides $r$. This moduli space admits a compactification which is a smooth DeligneMumford stack by allowing the bundles to degenerate over the nodes. There are also the tautological classes $\Psi_{i}$ associated to the cotangent line at the marked points $p_{i}$. When nonempty, the dimension of $\overline{\mathcal{M}}_{g, n}^{1 / r}(\boldsymbol{\alpha})$ is the same as that of $\overline{\mathcal{M}}_{g, n}$, namely $3 g-3+n$, since there are only a finite number of $r$-spin structures over a given stable curve. There is no analog in $r$-spin theory of the degree $d$ of a stable map into a manifold so we will ignore this parameter.

The virtual fundamental class in Gromov-Witten theory is replaced by the virtual class $c^{1 / r}$, a cohomology class in $H^{2 D}\left(\overline{\mathcal{M}}_{g, n}^{1 / r}(\boldsymbol{\alpha})\right)$ where $D=\frac{1}{r}\left((r-2)(g-1)+\sum_{i=1}^{n} \alpha_{i}\right)$. The analog of the cohomology ring of $M$ is the vector space $\mathcal{H}$ with a basis $\left\{\gamma_{0} \ldots \gamma_{r-2}\right\}$ with the pairing $\eta_{\alpha \beta}=\delta_{\alpha+\beta, r-2}$. The correlation functions are defined by

$$
\left\langle\tau_{n_{1}}\left(\gamma_{\alpha_{1}}\right) \tau_{n_{2}}\left(\gamma_{\alpha_{2}}\right) \ldots \tau_{n_{k}}\left(\gamma_{\alpha_{k}}\right)\right\rangle_{g}:=r^{1-g} \int_{\left.\overline{\mathcal{M}}_{g, k}^{1 / r}(\boldsymbol{\alpha})\right]} c^{1 / r} \cup \bigcup_{i=1}^{k} \Psi^{n_{i}}
$$

where $\left[\overline{\mathcal{M}}_{g, k}^{1 / r}(\boldsymbol{\alpha})\right]$ denotes the fundamental class of the stack $\overline{\mathcal{M}}_{g, k}^{1 / r}(\boldsymbol{\alpha})$.

As far as dimensional considerations are concerned, $r$-spin theory behaves as if it were Gromov-Witten theory of a manifold with real dimension $2(r-2) / r$ and $\gamma_{\alpha}$ behaves as if it were a cohomology class of dimension $2 \alpha / r$ for all $\alpha=0, \ldots, r-2$.

Applying Theorem 0.1 and dimensional arguments, we obtain the following.

Proposition 4.1 Let $r \geq 2$. In $r$-spin theory, the only possible nontrivial 1-point genus-3 correlators are as follows:

$r=2:\left\langle\tau_{7,0}\right\rangle_{3}=\frac{1}{82944}$

$r=3:\left\langle\tau_{6,1}\right\rangle_{3}=\frac{1}{31104}$

$r=4:\left\langle\tau_{6,0}\right\rangle_{3}=\frac{3}{20480}$

$r=5$ : All 1-point genus 3-correlators vanish for dimensional reasons.

$r=6:\left\langle\tau_{5,4}\right\rangle_{3}=\frac{2561}{20901888}$

$r \geq 7:\left\langle\tau_{5,4}\right\rangle_{3}$. Can be reduced to 5-point genus-0 correlators which can be calculated using the WDVV equation. 
Furthermore, when $r=3$, the only possible nontrivial 2-point correlators are

$$
\begin{aligned}
& \left\langle\tau_{7,0} \tau_{0,1}\right\rangle_{3}=1 / 15552, \quad\left\langle\tau_{6,0} \tau_{1,1}\right\rangle_{3}=19 / 77760, \quad\left\langle\tau_{5,0} \tau_{2,1}\right\rangle_{3}=47 / 77760, \\
& \left\langle\tau_{4,0} \tau_{3,1}\right\rangle_{3}=67 / 77760, \quad\left\langle\tau_{3,0} \tau_{4,1}\right\rangle_{3}=443 / 77760, \quad\left\langle\tau_{2,0} \tau_{5,1}\right\rangle_{3}=103 / 217728, \\
& \left\langle\tau_{1,0} \tau_{6,1}\right\rangle_{3}=5 / 31104, \quad\left\langle\tau_{0,0} \tau_{7,1}\right\rangle_{3}=1 / 31104 \text {. }
\end{aligned}
$$

Remark 4.2 When $r=3$, our results for $\left\langle\tau_{7,0} \tau_{0,1}\right\rangle_{3}$ and $\left\langle\tau_{6,1}\right\rangle_{3}$ both agree with the results of Shadrin [Sh] who calculated them by studying the geometry of $\overline{\mathcal{M}}_{3, n}^{1 / 3}$. He also showed that both of these numbers were consistent with the 3-rd KdV hierarchy as predicted by the generalized Witten conjecture.

\section{Appendix}

\section{A Gromov-Witten invariants of $\mathbb{C} P^{1}$ used to deter- mine the genus-3 topological recursion relation}

We need the following Gromov-Witten invariants of $\mathbb{C} P^{1}$ which are obtained using Gathmann's program based on the Virasoro constraints.

Genus-1 invariants:

$$
\left\langle\tau_{1,1}^{2}\right\rangle_{1,1}=\left\langle\tau_{3,0}\right\rangle_{1,1}=0, \quad\left\langle\tau_{1,1} \tau_{2,0}\right\rangle_{1,1}=1 / 8, \quad\left\langle\tau_{2,0}^{2}\right\rangle_{1,1}=-1 / 6, \quad\left\langle\tau_{2,1}\right\rangle_{1,1}=1 / 24 .
$$

Genus-2 invariants:

$$
\begin{array}{lll}
\left\langle\tau_{1,1}^{2}\right\rangle_{2,0}=0, & \left\langle\tau_{1,1} \tau_{2,0}\right\rangle_{2,0}=7 / 1920, & \left\langle\tau_{2,0}^{2}\right\rangle_{2,0}=-5 / 288, \\
\left\langle\tau_{2,1}\right\rangle_{2,0}=7 / 5760, & \left\langle\tau_{3,0}\right\rangle_{2,0}=-1 / 240, & \left\langle\tau_{1,1}^{4}\right\rangle_{2,1}=0, \\
\left\langle\tau_{1,1}^{3} \tau_{2,0}\right\rangle_{2,1}=0, & \left\langle\tau_{1,1}^{2} \tau_{2,0}^{2}\right\rangle_{2,1}=1 / 32, & \left\langle\tau_{1,1} \tau_{2,0}^{3}\right\rangle_{2,1}=17 / 64, \\
\left\langle\tau_{2,0}^{4}\right\rangle_{2,1}=5 / 6, & \left\langle\tau_{1,1}^{2} \tau_{2,1}\right\rangle_{2,1}=0, & \left\langle\tau_{1,1} \tau_{2,0} \tau_{2,1}\right\rangle_{2,1}=1 / 192, \\
\left\langle\tau_{2,0}^{2} \tau_{2,1}\right\rangle_{2,1}=23 / 576, & \left\langle\tau_{2,1}^{2}\right\rangle_{2,1}=1 / 576, & \left\langle\tau_{1,1}^{2} \tau_{3,0}\right\rangle_{2,1}=0, \\
\left\langle\tau_{1,1} \tau_{2,0} \tau_{3,0}\right\rangle_{2,1}=1 / 32, & \left\langle\tau_{2,0}^{2} \tau_{3,0}\right\rangle_{2,1}=11 / 96, & \left\langle\tau_{2,1} \tau_{3,0}\right\rangle_{2,1}=1 / 192, \\
\left\langle\tau_{3,0}^{2}\right\rangle_{2,1}=29 / 1440, & \left\langle\tau_{1,1} \tau_{3,1}\right\rangle_{2,1}=0, & \left\langle\tau_{2,0} \tau_{3,1}\right\rangle_{2,1}=1 / 192, \\
\left\langle\tau_{1,1} \tau_{4,0}\right\rangle_{2,1}=1 / 384, & \left\langle\tau_{2,0} \tau_{4,0}\right\rangle_{2,1}=41 / 2880, & \left\langle\tau_{4,1}\right\rangle_{2,1}=1 / 1920, \\
\left\langle\tau_{5,0}\right\rangle_{2,1}=1 / 576 . & &
\end{array}
$$


Genus-3 invariants

$$
\begin{array}{lll}
\left\langle\tau_{1,1}^{2} \tau_{2,1}\right\rangle_{3,0}=0, & \left\langle\tau_{1,1} \tau_{2,0} \tau_{2,1}\right\rangle_{3,0}=0, & \left\langle\tau_{2,0}^{2} \tau_{2,1}\right\rangle_{3,0}=-31 / 10752, \\
\left\langle\tau_{2,1}^{2}\right\rangle_{3,0}=0, & \left\langle\tau_{2,1} \tau_{3,0}\right\rangle_{3,0}=-31 / 96768, & \left\langle\tau_{3,0}^{2}\right\rangle_{3,0}=1501 / 725760, \\
\left\langle\tau_{1,1} \tau_{3,1}\right\rangle_{3,0}=0, & \left\langle\tau_{2,0} \tau_{3,1}\right\rangle_{3,0}=-31 / 96768, & \left\langle\tau_{1,1} \tau_{4,0}\right\rangle_{3,0}=-31 / 193536, \\
\left\langle\tau_{4,1}\right\rangle_{3,0}=-31 / 967680, & \left\langle\tau_{5,0}\right\rangle_{3,0}=41 / 290304, & \left\langle\tau_{3,1}^{2}\right\rangle_{3,1}=0, \\
\left\langle\tau_{1,1} \tau_{2,1} \tau_{4,0}\right\rangle_{3,1}=1 / 9216, & \left\langle\tau_{3,1} \tau_{4,0}\right\rangle_{3,1}=1 / 9216, & \left\langle\tau_{2,0}^{2} \tau_{4,1}\right\rangle_{3,1}=7 / 5760, \\
\left\langle\tau_{2,1} \tau_{4,1}\right\rangle_{3,1}=1 / 46080, & \left\langle\tau_{3,0} \tau_{4,1}\right\rangle_{3,1}=1 / 9216, & \left\langle\tau_{2,1} \tau_{5,0}\right\rangle_{3,1}=19 / 138240, \\
\left\langle\tau_{1,1} \tau_{5,1}\right\rangle_{3,1}=0, & \left\langle\tau_{2,0} \tau_{5,1}\right\rangle_{3,1}=1 / 15360, & \left\langle\tau_{1,1} \tau_{6,0}\right\rangle_{3,1}=1 / 46080, \\
\left\langle\tau_{6,1}\right\rangle_{3,1}=1 / 322560 . & &
\end{array}
$$

All other invariants needed to determine the genus-3 topological recursion relation can be computed from the string, dilaton, divisor equations, and the 0-point invariants

$$
\langle\rangle_{0,1}=1, \quad\langle\rangle_{1,0}=0
$$

\section{References}

[AC] Arbarello, E., and Cornalba, M., Calculating cohomology groups of moduli spaces of curves via algebraic geometry, Inst. Hautes Études Sci. Publ. Math. No. 88 (1998), 97-127.

[AL] Arcara, D., and Lee, Y.-P., Tautological equation in $\bar{M}_{3,1}$ via invariance conjectures, math. $A G / 0503184$.

[BP] Belorousski, P. and Pandharipande, R., A descendent relation in genus 2, Ann. Scuola Norm. Sup. Pisa Cl. Sci. (4) 29 (2000) 171-191.

[DZ] Dubrovin, B., Zhang, Y., Bihamiltonian hierarchies in 2D topological field theory at one-loop approximation, Comm. Math. Phys. 198 (1998) 311 - 361.

[EHX] Eguchi, T., Hori, K., and Xiong, C., Quantum Cohomology and Virasoro Algebra, Phys. Lett. B402 (1997) 71-80.

[FP] Faber, C., and Pandharipande, R., Relative maps and tautological classes, J. Eur. Math. Soc. (JEMS) 7 (2005), no. 1, 13-49.

[Ga] Gathmann, A., Topological recursion relations and Gromov-Witten invariants in higher genus, (math.AG/0305361).

[Ge1] Getzler, E., Intersection theory on $\bar{M}_{1,4}$ and elliptic Gromov-Witten Invariants, J. Amer. Math. Soc. 10 (1997) 973-998

[Ge2] Getzler, E., Topological recursion relations in genus 2, Integrable systems and algebraic geometry (Kobe/Kyoto, 1997), 73-106. 
[Gi] Givental, A., Gromov-Witten invariants and quantization of quadratic hamiltonians, Moscow Mathematical Journal, v.1, no. 4 (2001), 551-568.

[GV] Graber, T. and Vakil, R., Relative virtual localization and vanishing of tautological classes on moduli spaces of curves, math.AG/0309227

[Io] Ionel, E. Topological recursive relations in $H^{2 g}\left(\mathcal{M}_{g, n}\right)$. Invent. Math. 148 (2002), no. 3, 627-658.

[JKV] Jarvis, T., Kimura, T. and Vaintrob, A., Moduli spaces of higher spiin curves and integrable hierarchies, Compositio Math. 126 (2001), no. 2, 157-212, math.AG/9905034.

[K] Kontsevich, M., Intersection theory on the moduli space of curves and the matrix airy function, Comm. Math. Phys., 147 (1992).

[LiT] Li, J. and Tian, G., Virtual moduli cycles and Gromov-Witten invariants of general symplectic manifolds, Topics in symplectic 4-manifolds (Irvine, CA, 1996), 47-83.

[L1] Liu, X., Quantum product on the big phase space and Virasoro conjecture, Advances in Mathematics 169 (2002), 313-375.

[L2] Liu, X., Relations among universal equations for Gromov-Witten invariants, C. Hertling and M. Marcolli (Eds), Aspects of Mathematics, A publication of MaxPlanck-Institute for mathematics, Bonn, pp 169 - 180, 2004. (math.DG/0301161)

[Sh] Shadrin, S., Geometry of meromorphic functions and intersections on moduli spaces of curves, Int. Math. Res. Not. 2003, no. 38, 2051-2094, math.AG/0209282.

[RT] Ruan, Y. and Tian, G., Higher genus symplectic invariants and sigma models coupled with gravity, Invent. Math. 130 (1997), 455-516.

[W] Witten, E., Two dimensional gravity and intersection theory on Moduli space, Surveys in Diff. Geom., 1 (1991), 243-310.

Takashi Kimura

Department of Mathematics and Statistics

111 Cummington St.

Boston University

Boston, MA 02215, USA

E-mail address: kimura@math.bu.edu

Xiaobo Liu

Department of Mathematics 
University of Notre Dame

Notre Dame, IN 46556, USA

E-mail address: xliu3@nd.edu 